\title{
Numerical Modelling of large-scale Dynamics of the White Sea
}

\author{
Ilya Chernov
}

Institute of Applied Math Research, Petrozavodsk, 185910, Russia

*Corresponding Author: chernov@krc.karelia.ru

Copyright (C)2013 Horizon Research Publishing All rights reserved.

\begin{abstract}
In the paper we describe the numerical model of large-scale hydro- and thermal dynamics of the White sea. The model is the model of the Polar ocean by prof. N.G. Iakovlev adapted for the shallow and small White sea. Based on the primitive equations and the finite elements, this model includes mature and effective ice sea model. We show that the numerical simulation agree well with the generally accepted three-dimensional large-scale circulation of the sea, including thermohaline fields, currents, ice drift and thickness distribution. Numerical experiments show that influence of atmospheric forcing perturbations on the dynamics of the sea is not drastic. Changes in air temperature to a few degrees lead to similar changes of the water temperature and only slightly change the distributions of the fields. Complete, no, and altering (no clouds at summer, total cover at winter) cloud cover also changes the main patterns only slightly. This also proves that the model is stable with respect to atmospheric forcing.
\end{abstract}

Keywords Numerical modeling, large-scale circulation, the White sea, sea ice dynamics

\section{Introduction}

The White sea is interesting oceanological object from a few points of view. Firstly, this sea has several unique properties. It is important for Russia [1], partly because it is completely inside russian borders, also because of numerous economical, ecological, oceanological etc problems and questions. It is the part of the Arctic ocean. Finally, many observations has been collected and can be used for verifying mathematical models. Mathematical and computer modeling if hydrodynamics, thermal dynamics, ecological system, and so on, of the sea allows squeezing maximum information from the observed data. Also modeling is able to reduce costs, evaluate hardly measured quantities, make a forecast, estimate influence of different factors, perform numerical experiments (cheap even if real experiment is impossible, extremely expensive, or demands too much time).

Rapid development of computers in the recent decades has made mathematical modeling much significantly more powerful. Beside rapid development of ocean modeling in general [2], thermohydrodynamical models of the White sea have been developed [3],[4], [1] (see references therein for detailed review of recent research of the White sea). Experience of taking peculiarities of different seas into account has been accumulated, numerical methods and the appropriate software has been developed (e.g. [5], [6] and references therein). Nonlinear interaction of dynamical processes is important for the White sea $([3,7])$; this makes complex hydrodynamical models of high resolution necessary, and thus demands efficient algorithms and computers.

Special hydrological mode with dominating tidal currents facilitates development and implementation of complex models: the sea system quickly "forgets" the initial state, usually poorly known.

The aim of this article is application of large-scale model of thermal and hydrodynamics of the Arctic ocean, developed in the Institute of Numerical Mathematics by prof. N.G. Iakovlev [8], to the White sea. The main differences between the two seas are the vertical and horizontal scales. Average depth of the White sea is $70 \mathrm{~m}$, with the maximal not more than $340 \mathrm{~m}$. This makes the bathymetry much more important. Being small, the sea demands fine spatial grid. For the Arctic ocean the step of $1^{\circ}$ is sufficient, while for the White sea we need to take about $0.1^{\circ}$. Thus the time step also needs to be small. The result is that the White sea model is calculated longer than the model for much bigger ocean. Another point is the fact that the White sea is semi-closed, i.e. it is connected with the Barents sea via the common liquid boundary and has no other liquid boundaries (beside river mouths). The tidal wave induced from the Barents sea is rather strong and tidal flows dominate in the sea. Changes of sea level due to tides can be very high in bays, so free sea surface is essential when modeling the sea. River runoff is rather high for this small sea, so it is a problem to provide conservation of salinity: interaction of fresh water runoff and saline water inflow from the Barents sea must be balanced, so that salinity be almost constant, as it is in reality. There is no ice in the sea during summer, which is rather rare property of Arctic seas.

There just a few complex three-dimensional models 
of large-scale dynamics of water and sea ice adapted for the White sea. Prof. E.V. Semenov's model [3, 4] developed in Oceanology Institute of Russian Academy of Sciences describes only warm time of the year (no ice) and works in the short run (a few days). Climatic model of prof. I.A. Neelov [1] is the only one we know. On the other hand, prof. N.G. Iakovlev's model of sea and sea ice has been used for modeling the Arctic ocean, compared with other models (AOMIP project), and proved to be relevant and reliable tool for modeling large water objects. Besides, it is always good to have a few models of the same object in order to compare them, determine strong sides and drawbacks, organize interaction of different models, and so on.

\section{Numerical model of the White sea}

The model of large-scale (horizontal steps are less than $10 \mathrm{~km}$ ) thermal and hydrodynamics of the White sea is based on the primitive equations. Sea ice is described as ensemble of multiple flakes of ice; there are 14 gradations of ice thickness (i.e. discrete distribution), for each gradation there are masses of ice and snow and compactness. Compactness is the relative area covered by ice, and also can be treated as the probability to find a flake of the given thickness. Mass of ice of different gradations changes due to melting and freezing and also due to ridging. Thermal dynamics of the sea ice is described by locally one-dimensional model, taking into account the fact that ice thickness is anyway much less than the area of the grid cell. Rheology of the sea ice as the twodimensional continuous media is also described. The numerical problem is obtained using the finite elements approach.

The problem is solved in natural variables, i.e. velocities, pressure (or level), thermohaline fields. Coordinates are longitude $x$, complement $y$ of latitude to $90^{\circ}$, and depth $z$ measured from the average level downstairs. The water area is put into the square $50 \times 50$ equidistant (in the spherical measure) points. Thus the horizontal steps of the grid are determined: $h_{x}=0.246^{\circ} \approx 10$ $\mathrm{km}, h_{y}=0.074^{\circ} \approx 8.25 \mathrm{~km}$. Only 851 point from 2500 points of the grid correspond to water. The vertical grid consists of 16 layers which are less thick near the surface. The liquid boundary of the model sea is the line $\left.y \approx 67^{\circ} 29^{\prime} \mathrm{N}\right)$. The time grid is regular, with step $0.1 \mathrm{hr}$. Stability imposes rough limitations on the time step.

Tides induced from the Barents sea are extremely important for the White sea. Tides are described as harmonic oscillation of the sea level. The level of the Barents sea is higher compared to the average level of the free surface of the White sea. The atmospheric forcing includes air pressure, temperature. and humidity, wind velocity, cloud cover, and precipitation. They are taken from the NCEP data [9]. The bathymetry is interpolated to the grid points. Boundary data are of the Dirichlet type and monthly mean. Rivers are described as fresh water straits with monthly mean water temperature; the current velocity at the liquid boundary is calculated from the monthly mean runoff [1]. At the moment four main rivers are taken into account: Northern
Dvina, Mezen, Onega, and Kem. We tried to take nine rivers into account, but the results were hardly better, while the balance of fresh water was disturbed. Four main rivers seems an optimal solution. Initial fields are constant, zero for velocities, and there is no ice. The general pattern of currents and thermohaline distributions appears in a few months.

The output of the software are montly-mean fields of current velocities, ice velocities, water temperature and salinity, ice and snow masses ans ice compactness with respect to the thickness gradations, and the sea level distribution. The software can save instant fields for given times. All outputs are converted to self-describing netCDF format.

\section{Numerical experiments}

Large scale dynamics of the White sea was simulated during 30 years in order to compare the calculated fields with the observed ones and to determine whether the large scale pattern of currents, thermohaline structure of water, dynamics of the sea ice. The Open Source software package GrADS was used for graphical representations of the data.

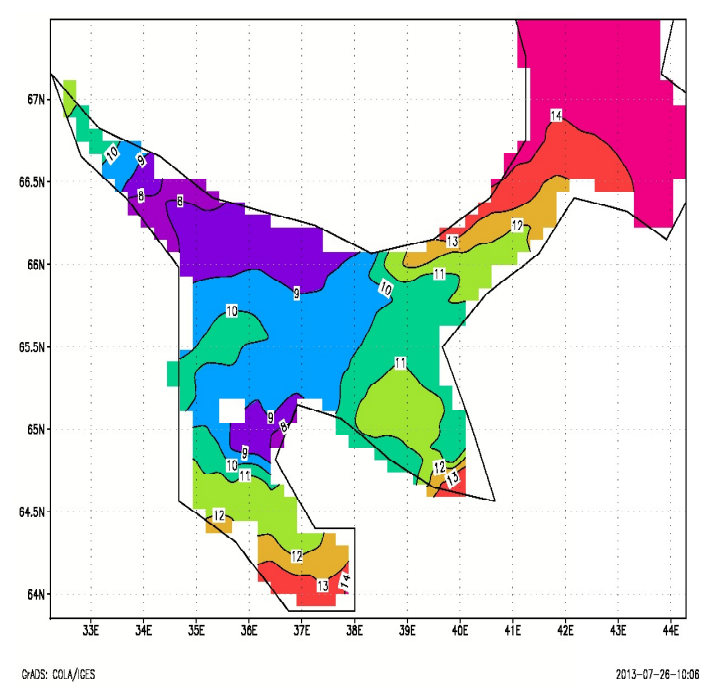

Figure 1. Spatial distribution of monthly-mean water temperature at the sea surface, august 1967, C.

In Figures 1 and 2 there are monthly mean temperature and salinity of water at the surface. For all years the pattern is generally the same, difference is due to wind and other variable factors and is not significant. The pattern coincide with observations and results obtained using other models [1]: water is warmer in the shallow bays, the Kelvin wave carrying salinity along the right shore from the Barents sea is represented, freshening effect of rivers is clearly seen, upwelling near Zhizhgin island is present, to mention a few.

Tidal currents dominate in the White sea $[3,1]$, though wind is able to change the pattern; however, in general it is conserved.

In Figure 3 there is the field of current velocity at the surface, for the month when the influence of wind was the least significant. The cyclonic circulation in the middle part of the Sea (the Basin) is clearly seen. This circulation is a part of quazi-geostrophical currents 


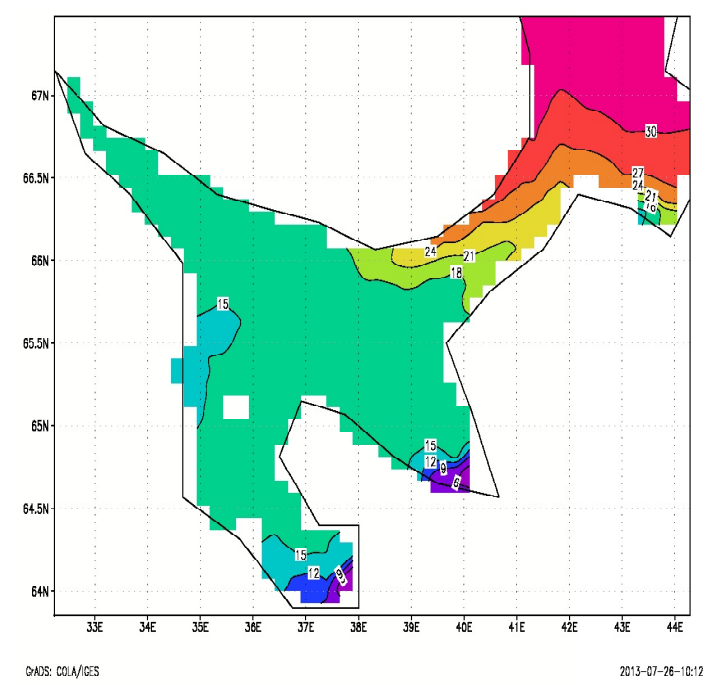

Figure 2. Spatial distribution of montly-mean water salinity at the sea surface, august 1967, per mille.

induced by the incoming tidal wave [3]. The obtained pattern agrees well with classical main flows (DeruginTimonov flows) present in the sea [1].

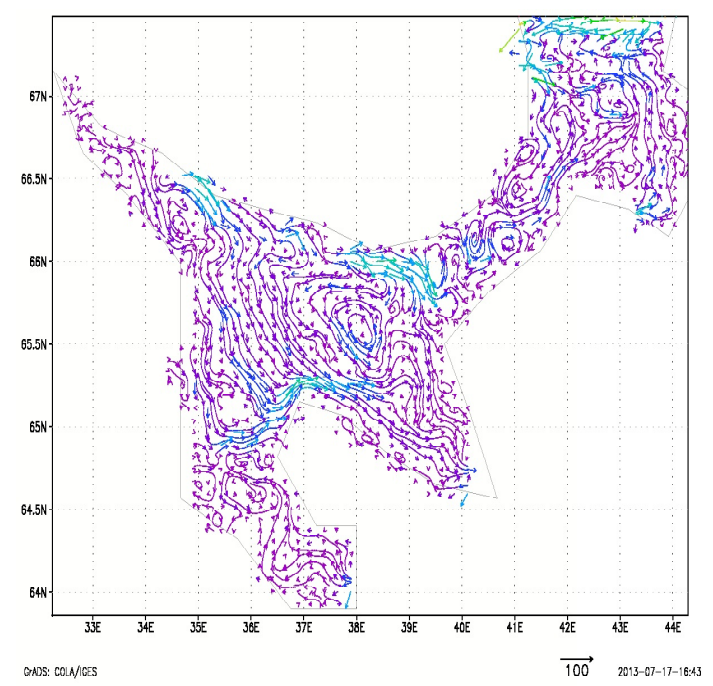

Figure 3. Spatial distribution of monthly-mean current velocity at the sea surface, january $1967, \mathrm{~cm} / \mathrm{s}$.

Ice thickness distribution Figure 4 coincides well with observations [1]: ice appears in December, by February most area of the sea is covered by ice. Ice is moved to the central and eastern regions of the sea. There is open water near the western shores. Strong ridges are in the funnel and the neck in the north-east part of the sea. In June ice completely disappears.

We tried to evaluate the influence of air temperature and cloud cover to the sea system. To do that we compared results of long run (twenty years) calculations of the model with original NCEP forcing with results for linearly perturbed forcing. In more detail, the air temperature near the water was increased by $1^{\circ} \mathrm{C}$, decreased by $1^{\circ} \mathrm{C}$, and increased during summer time (i.e. april-september) and decreased during winter time (i.e. october-march). Also the results for the real forcing were compared to those obtained for constantly $100 \%$ cloud cover, no clouds, and $100 \%$ during winter and no clouds during summer (such perturbation is called alter-

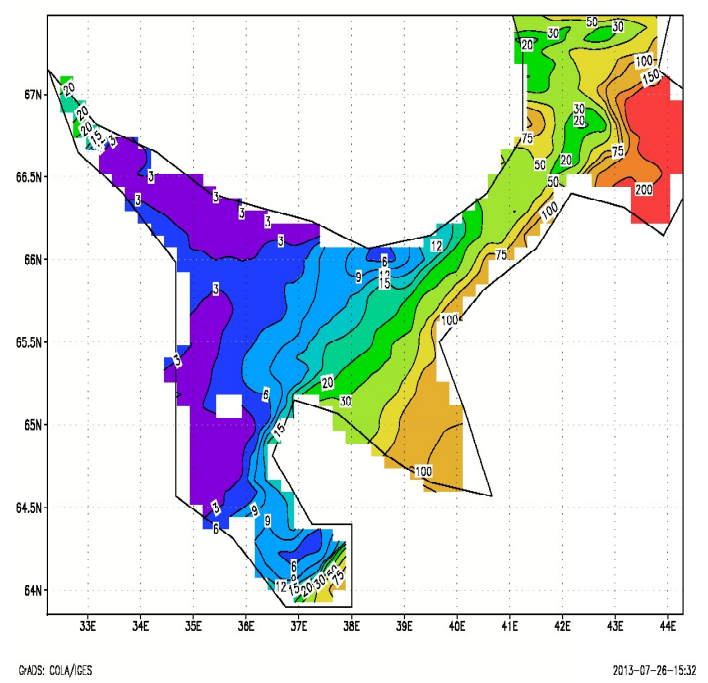

Figure 4. Spatial distribution of monthly-mean ice thickness at the sea surface, march $1967, \mathrm{~cm}$.

ing in this paper).

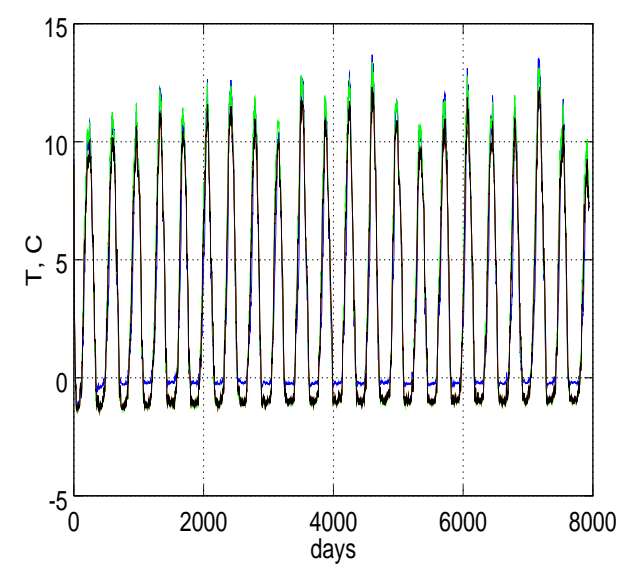

Figure 5. Daily mean average water temperature $(\mathrm{C})$ of the Sea with respect to time (days) for different regimes of cloud cover: blue is for the real cover from the NCEP reanalysis, green is $0 \%$ (no clouds), red is $100 \%$, black is no clouds in summer (aprilseptember), $100 \%$ clouds in winter.

The experiment showed that the model sea hardly reacts on such perturbations of the forcing. The contour lines of the fields vary only slightly, changes of both the average and extremal values of water temperature were $1{ }^{\circ} \mathrm{C}-2^{\circ} \mathrm{C}$. In Figure 5 there are daily mean surface water temperature of the sea for different cloud covers. Difference between $100 \%$ clouds (red line) and altering zerocomplete cloud cover (black line) is hardly noticeable, both no clouds and total cloud cover lead to slightly warmer sea. Beside the possible practical conclusion about stability of the sea system, with respect to climate change, we also can state that the model is stable with respect to unavoidable perturbations of the forcing.

The general conclusion is that the model has been successfully adopted for the conditions of the White sea and provides reasonable results with good coincidence with both observations and results for other models. In particular, such important phenomena as stationary up- 
welling, transport of water from the Barentz sea and rivers, main circulation pattern, and distribution of the sea ice thickness are represented in an acceptable way. The model is proven to be stable with respect to small perturbations of some atmospheric factors. However, tuning of the model in order to improve quantitative representation of the main fields is to be done. Also, the resolution obviously needs to be increased in order to describe narrow flows and warm and cold poles known to be present in the White sea.

\section{Acknowledgements}

The work has been financially supported by the grants 13-05-98802 and 13-05-12052 of the Russian foundation for basic research, and by the Program of strategic development of Petrozavodsk State University.

\section{REFERENCES}

[1] N. Filatov, D. Pozdnyakov, O. M. Johannessen, L. H. Pettersson, L. P. Bobylev. White Sea. Its Marine Environment and Ecosystem Dynamics Influenced by Global Change, Springer-Praxis, 2005.

[2] A. S. Sarkisyan. Fifty years of numerical modeling of baroclinic ocean, Izvestiya, Atmospheric and Oceanic Physics, Vol.48, Iss.1, 1-14.

[3] Semenov E. V. Numerical modeling of white sea dynamics and monitoring problem, Izvestiya, Atmo- spheric and Oceanic Physics, Vol.40, Iss.1, 114-126.

[4] Kasyanova N. A, Luneva M. V., Semenov E. V. Numerical modeling of white sea dynamics and monitoring problem, Izvestiya, Atmospheric and Oceanic Physics, Vol.37, Iss.6, 777-786.

[5] Ocean/Atmosphere Circulation Modeling Projects, Online available from http://stommel.tamu.edu/ ${ }^{\sim}$ baum/ocean_models.html.

[6] A. S. Sarkisyan, R. A. Ibrayev, N. G. Iakovlev. High resolution and four-dimensional analysis as a prospect for ocean modelling, Russian Journal of Numerical Analysis and Mathematical Modelling, Vol.25, Iss.5, 477-496.

[7] Zimin A. V. Internal waves on the White Sea shelf according to observations, Earth and Environmental Science. Oceanology, Vol.52, No.1, 11-20.

[8] N. G. Iakovlev. On the simulation of temperature and salinity fields in the Arctic Ocean, Izvestiya, Atmospheric and Oceanic Physics, Vol.48, Iss.1, 86-101.

[9] NCEP/NCAR Reanalysis, Online available from http://www.esrl.noaa.gov 